\title{
LITTLEWOOD-TYPE PROBLEMS ON SUBARCS OF THE UNIT CIRCLE
}

\author{
Peter Borwein and TAmás ERdélyi
}

Abstract. The results of this paper show that many types of polynomials cannot be small on subarcs of the unit circle in the complex plane. A typical result of the paper is the following. Let $\mathcal{F}_{n}$ denote the set of polynomials of degree at most $n$ with coefficients from $\{-1,0,1\}$. There are absolute constants $c_{1}>0, c_{2}>0$, and $c_{3}>0$ such that

$$
\exp \left(-c_{1} / a\right) \leq \inf _{0 \neq p \in \mathcal{F}_{n}}\|p\|_{L_{1}(A)}, \quad \inf _{0 \neq p \in \mathcal{F}_{n}}\|p\|_{A} \leq \exp \left(-c_{2} / a\right)
$$

for every subarc $A$ of the unit circle $\partial D:=\{z \in \mathbb{C}:|z|=1\}$ with length $0<a<c_{3}$.

The lower bound results extend to the class of $f$ of the form

$$
f(z)=\sum_{j=m}^{n} a_{j} z^{j}, \quad a_{j} \in \mathbb{C}, \quad\left|a_{j}\right| \leq M, \quad\left|a_{m}\right|=1
$$

with varying nonnegative integers $m \leq n$. It is shown that functions $f$ of the above form cannot be arbitrarily small uniformly on subarcs of the circle. However, this does not extend to sets of positive measure. It shown that it is possible to find a polynomial of the above form that is arbitrarily small on as much of the boundary (in the sense of linear Lebesgue measure) as one likes.

An easy to formulate corollary of the results of this paper is the following.

Corollary. Let $A$ be a subarc of the unit circle with length $\ell(A)=a$. If $\left(p_{k}\right)$ is a sequence of monic polynomials that tends to 0 in $L_{1}(A)$, then the sequence $H\left(p_{k}\right)$ of heights tends to $\infty$.

The results of this paper are dealing with (extensions of) classes much studied by Littlewood and many others in regards to the various conjectures of Littlewood concerning growth and flatness of unimodular polynomials on the unit circle $\partial D$. Hence the title of the paper.

1991 Mathematics Subject Classification. 11J54, $11 \mathrm{~B} 83$.

Key words and phrases. Transfinite diameter; integers; diophantine approximation; Chebyshev; polynomial; restricted coefficients; $-1,0,1$ coefficients; 0,1 coefficients.

Research is supported, in part, by the National Science Foundation of the USA under Grant No. DMS-9623156 and conducted while an International Postdoctoral Fellow of the Danish Research Council at University of Copenhagen (T. Erdélyi). Research is supported, in part, by NSERC of Canada (P. Borwein). 


\section{INTRODUCTION}

Littlewood's well-known and now resolved conjecture of around 1948 concerns polynomials of the form

$$
p(z):=\sum_{j=1}^{n} a_{j} z^{k_{j}},
$$

where the coefficients $a_{j}$ are complex numbers of modulus at least 1 and the exponents $k_{j}$ are distinct non-negative integers. It states that such polynomials have $L_{1}$ norms on the unit circle

$$
\partial D:=\{z \in \mathbb{C}:|z|=1\}
$$

that grow at least like $c \log n$ with an absolute constant $c>0$. This was proved by Konjagin [Ko-81] and independently by McGehee, Pigno, and Smith [MPS-81].

Pichorides, who contributed essentially to the proof of the Littlewood conjecture, observed in [Pi-83] that the original Littlewood conjecture (when all the coefficients are from $\{0,1\}$ would follow from a result on the $L_{1}$ norm of such polynomials on sets $E \subset \partial D$ of measure $\pi$. Namely if

$$
\int_{E}\left|\sum_{j=0}^{n} z^{k_{j}}\right||d z| \geq c
$$

for any subset $E \subset \partial D$ of measure $\pi$ with an absolute constant $c>0$, then the original Littlewood conjecture holds. Throughout the paper the measure of a set $E \subset \partial D$ is the linear Lebesgue measure of the set

$$
\left\{t \in[-\pi, \pi): e^{i t} \in E\right\} .
$$

Konjagin [Ko-96] gives a lovely probabilistic proof that this hypothesis fails. He does however conjecture the following: for any fixed set $E \subset \partial D$ of positive measure there exists a constant $c=c(E)>0$ depending only on $E$ such that

$$
\int_{E}\left|\sum_{j=0}^{n} z^{k_{j}}\right||d z| \geq c(E) .
$$

In other words the sets $E_{\epsilon} \subset \partial D$ of measure $\pi$ in his example where

$$
\int_{E_{\epsilon}}\left|\sum_{j=0}^{n} z^{k_{j}}\right||d z|<\epsilon
$$

must vary with $\epsilon>0$.

We show, among other things, that Konjagin's conjecture holds on subarcs of the unit circle $\partial D$.

Additional material on Littlewood's conjecture and related problems concerning the growth of polynomials with unimodular coefficients in various norms on the 
unit disk is to be found, for example, in [Bou-86], [Be-95], [Ka-85], [Li-86], [Ma-63], [Ne-90], [Od-93], and [So-95].

All the results of this paper concern how small polynomials of the above and related forms can be in the $L_{p}$ norms on subarcs of the unit disk. For $1 \leq p \leq \infty$ the results are sharp, at least up to a constant in the exponent.

An interesting related result is due to Nazarov [Na-93]. One of its simpler versions states that there is an absolute constant $c>0$ such that

$$
\max _{z \in I}|p(z)| \leq\left(\frac{c m(I)}{m(A)}\right)^{n} \max _{z \in A}|p(z)|
$$

for every polynomial $p$ of the form $p(z)=\sum_{j=0}^{n} a_{j} z^{k_{j}}$ with $k_{j} \in \mathbb{N}$ and $a_{j} \in \mathbb{C}$ and for every $A \subset I$, where $I$ is a subarc of $\partial D$ with length $m(I)$ and $A$ is measurable with Lebesgue measure $m(A)$. This extends a result of Turán [Tu-84] called Turán's Lemma, where $I=\partial D$ and $A$ is a subarc.

\section{Notation}

For $M>0$ and $\mu \geq 0$, let $\mathcal{S}_{M}^{\mu}$ denote the collection of all analytic functions $f$ on the open unit disk $D:=\{z \in \mathbb{C}:|z|<1\}$ that satisfy

$$
|f(z)| \leq \frac{M}{(1-|z|)^{\mu}}, \quad z \in D .
$$

We define the following subsets of $\mathcal{S}_{1}^{1}$. Let

$$
\mathcal{F}_{n}:=\left\{f: f(x)=\sum_{j=0}^{n} a_{j} x^{j}, a_{j} \in\{-1,0,1\}\right\}
$$

and denote the set of all polynomials with coefficients from the set $\{-1,0,1\}$ by

$$
\mathcal{F}:=\bigcup_{n=0}^{\infty} \mathcal{F}_{n}
$$

More generally we define the following classes of polynomials. For $M>0$ and $\mu \geq 0$ let

$$
\mathcal{K}_{M}^{\mu}:=\left\{f: f(x)=\sum_{j=0}^{n} a_{j} x^{j}, a_{j} \in \mathbb{C},\left|a_{j}\right| \leq M j^{\mu},\left|a_{0}\right|=1, n \in \mathbb{N}\right\} .
$$

On occasion we let $\mathcal{S}:=\mathcal{S}_{1}^{1}, \mathcal{S}_{M}:=\mathcal{S}_{M}^{1}$, and $\mathcal{K}_{M}:=\mathcal{K}_{M}^{0}$.

We also employ the following standard notations. We denote by $\mathcal{P}_{n}$ the set of all polynomials of degree at most $n$ with real coefficients. We denote by $\mathcal{P}_{n}^{c}$ the set of all polynomials of degree at most $n$ with complex coefficients. The height of a polynomial

$$
p_{n}(z):=\sum_{j=0}^{n} a_{j} z^{j}, \quad a_{j} \in \mathbb{C}, \quad a_{n} \neq 0
$$


is defined by

$$
H\left(p_{n}\right):=\max \left\{\frac{\left|a_{j}\right|}{\left|a_{n}\right|}: j=0,1, \ldots, n\right\} .
$$

Also,

$$
\|p\|_{A}:=\sup _{z \in A}|p(z)|
$$

and

$$
\|p\|_{L_{q}(A)}:=\left(\int_{A}|p(z)|^{q}|d z|\right)^{1 / q}
$$

are used throughout this paper for measurable functions (in this paper usually polynomials) $p$ defined on a measurable subset of the unit circle or the real line, and for $q \in(0, \infty)$.

\section{NeW Results}

The first two results concern lower bounds on subarcs in the supremum norm.

Theorem 3.1. Let $0<a<2 \pi$ and $M \geq 1$. Let $A$ be a subarc of the unit circle with length $\ell(A)=a$. Then there is an absolute constant $c_{1}>0$ such that

$$
\|f\|_{A} \geq \exp \left(\frac{-c_{1}(1+\log M)}{a}\right)
$$

for every $f \in \mathcal{S}_{M}\left(:=\mathcal{S}_{M}^{1}\right)$ that is continuous on the closed unit disk and satisfies $\left|f\left(z_{0}\right)\right| \geq \frac{1}{2}$ for every $z_{0} \in \mathbb{C}$ with $\left|z_{0}\right|=\frac{1}{4 M}$.

Corollary 3.2. Let $0<a<2 \pi$ and $M \geq 1$. Let $A$ be a subarc of the unit circle with length $\ell(A)=a$. Then there is an absolute constant $c_{1}>0$ such that

$$
\|f\|_{A} \geq \exp \left(\frac{-c_{1}(1+\log M)}{a}\right)
$$

for every $f \in \mathcal{K}_{M}\left(:=\mathcal{K}_{M}^{1}\right)$.

The next two results show that the previous results are, up to constants, sharp.

Theorem 3.3. Let $0<a<2 \pi$. Let $A$ be the subarc of the unit circle with length $\ell(A)=a$. Then there are absolute constants $c_{1}>0$ and $c_{2}>0$ such that

$$
\inf _{0 \neq f \in \mathcal{F}}\|f\|_{A} \leq \exp \left(\frac{-c_{1}}{a}\right)
$$

whenever $\ell(A)=a \leq c_{2}$. 
Theorem 3.4. Let $0<a<2 \pi$ and $M \geq 1$. Let $A$ be the subarc of the unit circle with length $\ell(A)=a$. Then there are absolute constants $c_{1}>0$ and $c_{2}>0$ such that

$$
\inf _{0 \neq f \in \mathcal{K}_{M}}\|f\|_{A} \leq \exp \left(\frac{-c_{1}(1+\log M)}{a}\right)
$$

whenever $\ell(A)=a \leq c_{2}$.

The next two results extend the first two results to the $L_{1}$ norm (and hence to all $L_{p}$ norms with $p \geq 1$ ).

Theorem 3.5. Let $0<a<2 \pi, M \geq 1$, and $\mu=1,2, \ldots$ Let $A$ be a subarc of the unit circle with length $\ell(A)=a$. Then there is an absolute constant $c_{1}>0$ such that

$$
\|f\|_{L_{1}(A)} \geq \exp \left(\frac{-c_{1}(\mu+\log M)}{a}\right)
$$

for every $f \in \mathcal{S}_{M}^{\mu}$ that is continuous on the closed unit disk and satisfies $\left|f\left(z_{0}\right)\right| \geq \frac{1}{2}$ for every $z_{0} \in \mathbb{C}$ with $\left|z_{0}\right| \leq \frac{1}{4 M 2^{\mu}}$.

Corollary 3.6. Let $0<a<2 \pi, M \geq 1$, and $\mu=1,2, \ldots$. Let $A$ be a subarc of the unit circle with length $\ell(A)=a$. Then there is an absolute constant $c_{1}>0$ such that

$$
\|f\|_{L_{1}(A)} \geq \exp \left(\frac{-c_{1}(1+\mu \log \mu+\log M)}{a}\right)
$$

for every $f \in \mathcal{K}_{M}^{\mu}$.

The following is an interesting consequence of the preceding results.

Corollary 3.7. Let $A$ be a subarc of the unit circle with length $\ell(A)=a$. If $\left(p_{k}\right)$ is a sequence of monic polynomials that tends to 0 in $L_{1}(A)$, then the sequence $H\left(p_{k}\right)$ of heights tends to $\infty$.

The final result shows that the theory does not extend to arbitrary sets of positive measure.

Theorem 3.8. For every $\epsilon>0$ there is a polynomial $p \in \mathcal{K}_{1}$ such that $|p(z)|<\epsilon$ everywhere on the unit circle except possibly in a set of linear measure at most $\epsilon$.

The above results should be compared with earlier result of the authors [Bor96] on approximation on the interval $[0,1]$. These state that there are absolute constants $c_{1}>0$ and $c_{2}>0$ such that

$$
\exp \left(-c_{1} \sqrt{n}\right) \leq \inf _{0 \neq p \in \mathcal{F}_{n}}\|p\|_{[0,1]} \leq \exp \left(-c_{2} \sqrt{n}\right)
$$

for every $n \geq 2$. 


\section{LEMMAS}

Lemma 4.1. Let $0<a<\pi$ and $M \geq 1$. Let $\Gamma_{M}$ be the circle with diameter $\left[-1+\frac{1}{2 M}, 1\right]$. Let $J$ be the subarc of $\Gamma_{M}$ with length $\ell(J)=$ a which is symmetric with respect to the real line and contains 1 . Then there is an absolute constant $c_{3}>0$ such that

$$
\|g\|_{J} \geq \exp \left(\frac{-c_{3}(1+\log M)}{a}\right)
$$

for all $g \in \mathcal{S}_{4 M}$ that is continuous on the closed unit disk and satisfies $\left|g\left(\frac{1}{4 M}\right)\right| \geq \frac{1}{4}$.

Our next lemma is known as (a version) of the three-line-theorem. It may be found, for example, in [Zy-59, p. 93]. Its proof is so short and simple that we present it for the sake of completeness.

Lemma 4.2. Let $a>0$ and

$$
E_{a}:=\{z \in \mathbb{C}: 0 \leq \operatorname{Im}(z) \leq a\} .
$$

Suppose $g$ is an analytic function in the interior of $E_{a}$, and suppose $g$ is continuous on $E_{a} \cup \infty$. Then

$$
\max _{\{z: \operatorname{Im}(z)=a / 2\}}|g(z)| \leq\left(\max _{\{z: \operatorname{Im}(z)=0\}}|g(z)|\right)^{1 / 2}\left(\max _{\{z: \operatorname{Im}(z)=a\}}|g(z)|\right)^{1 / 2} .
$$

The next lemma, that plays a crucial role in the proof of Theorem 3.1, can be easily derived from Lemma 4.2 .

Lemma 4.3. Let $0<a<\pi, \alpha:=\cos (a / 2)+i \sin (a / 2), \beta:=\cos (a / 2)-i \sin (a / 2)$. Let

$$
I_{t}:=\left\{z \in \mathbb{C}: \arg \left(\frac{\alpha-z}{z-\beta}\right)=t\right\} .
$$

Note that $I_{a}$ is the smaller arc on the unit circle with endpoints $\alpha$ and $\beta$, and $I_{0}$ is the line segment between $\alpha$ and $\beta$. Suppose $g$ is an analytic function in the open region bounded by $I_{0}$ and $I_{a}$, and suppose $g$ is continuous on the closed region between $I_{0}$ and $I_{a}$. Then

$$
\max _{z \in I_{a / 2}}|g(z)| \leq\left(\max _{z \in I_{0}}|g(z)|\right)^{1 / 2}\left(\max _{z \in I_{a}}|g(z)|\right)^{1 / 2} .
$$

To prove Theorem 3.3, we need some corollaries of the

Hadamard Three Circles Theorem. Suppose $f$ is regular inside and on

$$
\left\{z \in \mathbb{C}: r_{1} \leq|z| \leq r_{2}\right\} \text {. }
$$

For $r \in\left[r_{1}, r_{2}\right]$, let

$$
M(r):=\max _{|z|=r}|f(z)|
$$

Then

$$
M(r)^{\log \left(r_{2} / r_{1}\right)} \leq M\left(r_{1}\right)^{\log \left(r_{2} / r\right)} M\left(r_{2}\right)^{\log \left(r / r_{1}\right)}
$$


Corollary 4.4. Let $a \in(0,1 / 8]$. Suppose $f$ is regular inside and on the ellipse $E_{a}$ with foci at $1-8 a$ and 1 and with major axis

$$
[(1-4 a)-17 a,(1-4 a)+17 a] .
$$

Let $\widetilde{E}_{a}$ be the ellipse with foci at $1-a$ and 1 and with major axis

$$
[(1-4 a)-10 a,(1-4 a)+10 a] .
$$

Then

$$
\max _{z \in \widetilde{E}_{a}}|f(z)| \leq\left(\max _{z \in[1-8 a, 1]}|f(z)|\right)^{1 / 2}\left(\max _{z \in E_{a}}|f(z)|\right)^{1 / 2} .
$$

Corollary 4.5. We have

$$
\max _{z \in \widetilde{E}_{a}}|f(z)| \leq((n+1) \exp (13 n a))^{1 / 2}\left(\max _{z \in[1-8 a, 1]}|f(z)|\right)^{1 / 2}
$$

for every $f \in \mathcal{F}_{n}$ and $a \in(0,1 / 8]$.

In one of our proofs of Theorem 3.3 we will need the upper bound of the result below proved in [Bor-96]. An application of this lemma makes the proof of Theorem 3.3 shorter in the special case when the subarc $A$ of the unit circle is symmetric with respect to the real line and contains 1 . Our second proof of Theorem 3.3 is longer. This self-contained second proof does not use the lemma below and eliminates the extra assumption on the subarc $A$.

Lemma 4.6. There are absolute constants $c_{4}>0$ and $c_{5}>0$ such that

$$
\exp \left(-c_{4} \sqrt{n}\right) \leq \inf _{0 \neq p \in \mathcal{F}_{n}}\|p\|_{[0,1]} \leq \exp \left(-c_{5} \sqrt{n}\right)
$$

for every $n \geq 2$.

To prove Theorem 3.4 we need two lemmas.

Lemma 4.7. Suppose

$$
\begin{aligned}
& p(x)=\sum_{j=0}^{n} a_{j} x^{j}, \quad\left|a_{j}\right| \leq 9, \quad a_{j} \in \mathbb{C}, \\
& p(x)=(x-1)^{k} q(x), \quad q(x)=\sum_{j=0}^{n-k} b_{j} x^{j}, \quad b_{j} \in \mathbb{C} .
\end{aligned}
$$

Then

$$
\|q\|_{\partial D} \leq \sum_{j=0}^{n-k}\left|b_{j}\right| \leq 9(n+1) e\left(\frac{e n}{k}\right)^{k-1} \leq 9(n+1)\left(\frac{e n}{k}\right)^{k}
$$

(where $\partial D$ denotes the unit circle). As a consequence, if $A$ denotes the subarc of the unit circle that is symmetric to the real line, contains 1 , and has length $2 k /(9 n)$, then

$$
\|p\|_{A} \leq 9(n+1)\left(\frac{e}{9}\right)^{k}
$$

To prove Theorem 3.4 our main tool is the next lemma due to Halász [Tu-84]. 
Lemma 4.8. For every $k \in \mathbb{N}$, there exists a polynomial $h \in \mathcal{P}_{k}$ such that

$$
h(0)=1, \quad h(1)=0, \quad|h(z)|<\exp \left(\frac{2}{k}\right) \quad \text { for } \quad|z| \leq 1 .
$$

Lemma 4.9. Let $0<a<\pi$ and $M \geq 1$. Let $\Gamma_{a, M, \mu}$ be the circle with diameter $\left[-1+\frac{1}{2 M 2^{\mu}}, 1-\frac{1-\cos (a / 4)}{M 2^{\mu}}\right]$. Let $I$ be the subarc of $\Gamma_{a, M, \mu}$ with length $\ell(I)=a$ which is symmetric with respect to the real line and contains $1-\frac{1-\cos (a / 4)}{M 2^{\mu}}$. Then there is an absolute constant $c_{4}>0$ such that

$$
\|g\|_{I} \geq \exp \left(\frac{-c_{4}(\mu+\log M)}{a}\right)
$$

for every $g \in \mathcal{S}_{M 4^{\mu}}^{\mu}$ that satisfies

$$
\left|g\left(\frac{1}{4 M 2^{\mu}}-\frac{1-\cos (a / 4)}{2 M 2^{\mu}}\right)\right| \geq \frac{1}{4} .
$$

Lemma 4.10. Let $w_{1} \neq w_{2} \in \mathbb{C}$ and let $z_{0}:=\frac{1}{2}\left(w_{1}+w_{2}\right)$. Assume that $J_{1}$ is an arc that connects $w_{1}$ and $w_{2}$. Let $J_{2}$ be the arc that is the symmetric image of $J_{1}$ with respect to the $z_{0}$. Let $J:=J_{1} \cup J_{2}$ be positively oriented. Suppose that $g$ is an analytic function inside and on $J$. Suppose that the region inside $J$ contains the disk centered at $z_{0}$ with radius $\delta>0$. Let $|g(z)| \leq K$ for $z \in J_{2}$. Then

$$
\left|g\left(z_{0}\right)\right|^{2} \leq(\pi \delta)^{-1} K \int_{J_{1}}|g(z)||d z| .
$$

\section{Proofs of Theorems $3.1-3.6$}

Proof of Lemma 4.1. Suppose $g \in \mathcal{S}_{4 M}$ is continuous on the closed unit disk and $\left|g\left(\frac{1}{4 M}\right)\right| \geq \frac{1}{4}$. Let $2 m \geq 4$ be the smallest even integer not less than $4 \pi / a$. Let

$$
\xi:=\exp \left(\frac{2 \pi i}{2 m}\right)
$$

be the first $(2 m)$ th root of unity. We define $2 m$ equally spaced points on $\Gamma_{M}$ by

$$
\eta_{k}:=\frac{1}{4 M}+\left(1-\frac{1}{4 M}\right) \xi^{k}, \quad k=0,1, \ldots, 2 m-1 .
$$

Then there is an absolute constant $c_{5}>0$ such that

$$
1-|z| \geq c_{5} M^{-1}(k a)^{2}, \quad k=1,2, \ldots m-1,
$$

whenever $z$ is on the smaller subarc of the circle $\Gamma_{M}$ with endpoints $\eta_{k}$ and $\eta_{k+1}$ or with endpoints $\eta_{2 m-k}$ and $\eta_{2 m-k-1}$, respectively. We define the function

$$
h(z):=\prod_{j=0}^{2 m-1} g\left(\frac{1}{4 M}+\left(1-\frac{1}{4 M}\right) \xi^{j}\left(z-\frac{1}{4 M}\right)\right) .
$$


Since $g \in \mathcal{S}_{4 M}$, we obtain

$$
\begin{aligned}
\max _{z \in \Gamma_{M}}|h(z)| & \leq\|g\|_{J}^{2} \prod_{k=1}^{m-1}\left(4 M\left(c_{4} M^{-1}(k a)^{2}\right)^{-1}\right)^{2} \leq\left(\frac{2}{c_{5} a}\right)^{4 m-4} \frac{M^{4 m-4}}{((m-1) !)^{4}}\|g\|_{J}^{2} \\
& \leq\left(\frac{m}{\pi c_{5}}\right)^{4 m-4}\left(\frac{M e}{m-1}\right)^{4 m-4}\|g\|_{J}^{2} \leq e^{4}\left(\frac{e M}{\pi c_{5}}\right)^{4 m-4}\|g\|_{J}^{2} \\
& \leq \exp \left(\frac{c_{6}(1+\log M)}{a}\right)\|g\|_{J}^{2}
\end{aligned}
$$

with an absolute constant $c_{6}>0$. Now the Maximum Principle yields that

$$
\left|g\left(\frac{1}{4 M}\right)\right|^{2 m}=\left|h\left(\frac{1}{4 M}\right)\right| \leq \max _{z \in \Gamma_{M}}|h(z)| \leq \exp \left(\frac{c_{6}(1+\log M)}{a}\right)\|g\|_{J}^{2} .
$$

Since $2 m \leq 2+4 \pi / a$ and $\left|g\left(\frac{1}{4 M}\right)\right| \geq \frac{1}{4}$, we obtain

$$
\begin{aligned}
\|g\|_{J}^{2} & \geq \exp \left(\frac{-c_{6}(1+\log M)}{a}\right)\left|g\left(\frac{1}{4 M}\right)\right|^{2 m} \geq \exp \left(\frac{-c_{6}(1+\log M)}{a}\right)\left(\frac{1}{4}\right)^{2 m} \\
& \geq \exp \left(\frac{-c_{7}(1+\log M)}{a}\right)
\end{aligned}
$$

with an absolute constant $c_{7}>0$. This finishes the proof.

Proof of Lemma 4.2. Let $z_{0}$ be a complex number with $\operatorname{Im}(z)=a / 2$. We want to show that

$$
\left|g\left(z_{0}\right)\right|^{2} \leq\left(\max _{\{z: \operatorname{Im}(z)=0\}}|g(z)|\right)\left(\max _{\{z: \operatorname{Im}(z)=a\}}|g(z)|\right) .
$$

Without loss of generality we may assume that $\operatorname{Re}\left(z_{0}\right)=0$; the general case follows by a linear transformation. So let $z_{0}:=i a / 2$. Applying the Maximum Principle to $h(z):=g(z) g(i a-z)$ on $E_{a} \cup \infty$, we obtain

$$
\begin{aligned}
|g(i a / 2)|^{2} & =|g(i a / 2) g(i a-i a / 2)|=|h(i a / 2)| \leq \max _{z \in \partial E_{a}}|h(z)| \\
& =\max _{z \in \partial E_{a}}|g(z) g(i a-z)| \leq\left(\max _{\{z: \operatorname{Im}(z)=0\}}|g(z)|\right)\left(\max _{\{z: \operatorname{Im}(z)=a\}}|g(z)|\right) .
\end{aligned}
$$

and the proof is finished.

Proof of Lemma 4.3. This follows from Lemma 4.2 by the substitution

$$
w:=\log \left(\frac{\alpha-z}{z-\beta}\right) .
$$

Proof of Theorem 3.1. Without loss of generality we may assume that the $\operatorname{arc} A$ is the subarc of the unit circle with length $\ell(A)=a<\pi$ which is symmetric with respect to the real line and contains 1. Suppose $f \in \mathcal{S}_{M}$ is continuous on the 
open unit disk, and suppose $\left|f\left(\frac{1}{4 M}\right)\right| \geq \frac{1}{2}$. Using the notation of Lemma 4.3, let $g(z):=(z-\alpha)(z-\beta) f(z)$. Then a straightforward geometric argument yields that

$$
|g(z)| \leq \frac{M|(z-\alpha)(z-\beta)|}{1-|z|} \leq \frac{2 M}{\sin (a / 2)}, \quad z \in I_{0}
$$

(note that $I_{0}$ is the line segment between $\alpha$ and $\beta$ ). Hence, with $L:=\|g\|_{A}$ (note that $A=I_{a}$ ), we conclude by Lemma 4.3 that

$$
\max _{z \in I_{a / 2}}|g(z)| \leq\left(\frac{2 M L}{\sin (a / 2)}\right)^{1 / 2}
$$

Denote by $G_{a}$ the open region bounded by $I_{a / 2}$ and $I_{a}$. By the Maximum Principle

$$
\max _{z \in G_{a}}|g(z)| \leq \max \left\{L,\left(\frac{2 L}{\sin (a / 2)}\right)^{1 / 2}\right\}
$$

It is a simple elementary geometry to show that the arc $K:=\Gamma_{M} \cap G_{a}$ has length at least $a / 2$. Here, as in Lemma $4.1, \Gamma_{M}$ denotes the circle with diameter $\left[-1+\frac{1}{2 M}, 1\right]$. Observe that $f \in \mathcal{S}_{M}$ implies $g \in \mathcal{S}_{4 M}$. Also, since $M \geq 1$,

$$
\left|g\left(\frac{1}{4 M}\right)\right| \geq\left(\frac{3}{4}\right)^{2}\left|f\left(\frac{1}{4 M}\right)\right| \geq\left(\frac{3}{4}\right)^{2} \frac{1}{2} \geq \frac{1}{4}
$$

Hence Lemma 4.1 can be applied with $g \in \mathcal{S}_{4 M}$. We conclude that

$$
\max \left\{L,\left(\frac{2 M L}{\sin (a / 2)}\right)^{1 / 2}\right\} \geq\|g\|_{K} \geq \exp \left(\frac{-c_{4}(1+\log M)}{2 a}\right)
$$

from which

$$
\|f\|_{A} \geq \frac{1}{4}\|g\|_{A}=\frac{L}{4} \geq \exp \left(\frac{-c_{1}(1+\log M)}{a}\right)
$$

with an absolute constant $c_{1}>0$.

Proof of Corollary 3.2. Note that if $f \in \mathcal{K}_{M}$, then $f \in \mathcal{S}_{M}$ and $f$ is continuous on the closed unit disk. Also, if $\left|z_{0}\right|=\frac{1}{4 M}$, then

$$
\mid f\left(z_{0} \mid \geq 1-M \frac{\left|z_{0}\right|}{1-\left|z_{0}\right|} \geq 1-\frac{2 M}{4 M}=\frac{1}{2} .\right.
$$

So the assumptions of Theorem 3.1 are satisfied and the corollary follows from Theorem 3.1.

Proof of Corollary 4.4. This follows from the Hadamard Three Circles Theorem with the substitution

$$
w=(1-4 a)+4 a\left(\frac{z+z^{-1}}{2}\right)
$$


The Hadamard Three Circles Theorem is applied with $r_{1}:=1, r:=2$, and $r_{2}:=$ 4.

Proof of Corollary 4.5. This follows from Corollary 4.4 and the Maximum Principle.

We present two proofs of Theorem 3.3. The first one is under the additional assumption that the subarc $A$ is symmetric with respect to the real line and contains 1.

Proof of Theorem 3.3 in the above special case. By Lemma 4.6, for every integer $n \geq 2$, there is a $Q_{n} \in \mathcal{F}_{n}$ such that

$$
\left\|Q_{n}\right\|_{[0,1]} \leq \exp \left(-c_{5} \sqrt{n}\right) \text {. }
$$

Let $n$ be chosen so that $n:=\lfloor N\rfloor$, where $N$ is defined by

$$
a=\frac{c_{5}}{26 \sqrt{N}} .
$$

Then, by Corollary 4.5,

$$
\begin{aligned}
\max _{z \in \widetilde{E}_{a}}\left|Q_{n}(z)\right| & \leq(n+1)^{1 / 2}\left(\exp \left(\left(c_{5} / 2\right) \sqrt{n}\right)\right)^{1 / 2}\left(\max _{z \in[1-8 a, 1]}|f(z)|\right)^{1 / 2} \\
& \leq(n+1)^{1 / 2}\left(\exp \left(\left(c_{5} / 2\right) \sqrt{n}\right)\right)^{1 / 2}\left(\exp \left(-c_{5} \sqrt{n}\right)\right)^{1 / 2} \\
& \leq(n+1)^{1 / 2} \exp \left(-\left(c_{5} / 4\right) \sqrt{n}\right) \\
& \leq \exp \left(\frac{-c_{6}}{a}\right)
\end{aligned}
$$

whenever $a \leq c_{7}$, where $c_{6}>0$ and $c_{7}>0$ are absolute constants. Now observe that the unit circle intersects the ellipse $\widetilde{E}_{a}$ in an arc of length at least $c_{8} a$, where $c_{8}>0$ is an absolute constant. Therefore the Maximum Principle finishes the proof.

Our second proof of Theorem 3.3 is in the general case. In addition, it is selfcontained.

Proof of Theorem 3.3. Let $A:=\left\{e^{i t}: t \in\left[t_{1}, t_{2}\right]\right\}$, where $0 \leq t_{1}<t_{2} \leq 2 \pi$ and $t_{1}-t_{2}=a$. Let $t_{0}:=\left(t_{1}+t_{2}\right) / 2$, and $w:=e^{i t_{0}}$. We prove the following extension of Lemma 4.6. For every sufficiently large $n \in \mathbb{N}$, there is a $Q_{n} \in \mathcal{F}_{n}$ such that

$$
\left\|Q_{n}\right\|_{[0, w]} \leq \exp \left(-c_{5} \sqrt{n}\right) .
$$

To see this we define $k:=\left\lfloor\frac{1}{2} \sqrt{n}\right\rfloor$. Let

$$
1-k /(2 n)=: y_{0}<y_{1}<\cdots<y_{k}:=1
$$

be $k+1$ equidistant points. 
We use a counting argument to find a polynomial $f \in \mathcal{F}_{n-1}$ with the property

$$
\left|f\left(y_{j} w\right)\right| \leq 2^{1-\sqrt{n}}, \quad j=0,1, \ldots, k,
$$

for sufficiently large $n$. Indeed, we can divide the $2(k+1)$-dimensional real cube

$$
Q:=\left\{\left(z_{0}, \ldots, z_{k}\right) \in \mathbb{C}^{k}: \operatorname{Re}\left(z_{j}\right), \operatorname{Im}\left(z_{j}\right) \in[-n-1, n+1)\right\}
$$

into $(2 m(n+1))^{2(k+1)}$ subcubes by defining

$$
\begin{gathered}
Q_{i_{0}, i_{1}, \ldots, i_{2 k+1}} \\
:=\left\{\left(z_{0}, \ldots, z_{k}\right) \in \mathbb{C}^{k}: \operatorname{Re}\left(z_{j}\right) \in\left[\frac{i_{2 j}}{m}, \frac{i_{2 j}+1}{m}\right), \operatorname{Im}\left(z_{j}\right) \in\left[\frac{i_{2 j+1}}{m}, \frac{i_{2 j+1}+1}{m}\right)\right\},
\end{gathered}
$$

where $\left(i_{0}, i_{1}, \ldots, i_{2 k+1}\right)$ are $2(k+1)$-tuples of integers with $-(n+1) m \leq i_{j} \leq$ $(n+1) m-1$ for each $j=0,1, \ldots, 2 k+1$. Let

$$
\mathcal{A}_{n-1}:=\left\{f: f(x)=\sum_{j=0}^{n-1} a_{j} x^{j}, a_{j} \in\{0,1\}\right\}
$$

denote the set of polynomials of degree at most $n-1$ with coefficients from $\{0,1\}$. Note that if $P \in \mathcal{A}_{n-1}$, then

$$
M(P):=\left(\operatorname{Re}\left(P\left(y_{0} w\right)\right), \operatorname{Im}\left(P\left(y_{0} w\right)\right), \ldots, \operatorname{Re}\left(P\left(y_{k} w\right)\right), \operatorname{Im}\left(P\left(y_{k} w\right)\right)\right) \in Q .
$$

Also, there are exactly $2^{n}$ elements of $\mathcal{A}_{n-1}$. Therefore, if

$$
(2 m(n+1))^{2(k+1)}<2^{n}
$$

holds, then there exist two different $P_{1} \in \mathcal{A}_{n-1}$ and $P_{2} \in \mathcal{A}_{n-1}$, and a subcube $Q_{i_{0}, i_{1}, \ldots, i_{2 k+1}}$ such that both

$$
M\left(P_{1}\right)=\left(\operatorname{Re}\left(P_{1}\left(y_{0} w\right)\right), \operatorname{Im}\left(P_{1}\left(y_{0} w\right)\right), \ldots, \operatorname{Re}\left(P_{1}\left(y_{k} w\right)\right), \operatorname{Im}\left(P_{1}\left(y_{k} w\right)\right)\right)
$$

and

$$
M\left(P_{2}\right)=\left(\operatorname{Re}\left(P_{2}\left(y_{0} w\right)\right), \operatorname{Im}\left(P_{2}\left(y_{0} w\right)\right), \ldots, \operatorname{Re}\left(P_{2}\left(y_{k} w\right)\right), \operatorname{Im}\left(P_{2}\left(y_{k} w\right)\right)\right)
$$

are in $Q_{i_{0}, i_{1}, \ldots, i_{2 k+1}}$, and hence for $0 \neq f:=P_{1}-P_{2} \in \mathcal{F}_{n-1}$, we have

$$
\left|f\left(y_{j} w\right)\right| \leq \sqrt{2} m^{-1}, \quad j=0,1, \ldots, k .
$$

Now choose $m:=\left\lfloor 2^{-\sqrt{n}}\right\rfloor$. This, together with $k:=\left\lfloor\frac{1}{2} \sqrt{n}\right\rfloor$, yields that the inequality $(2 m n)^{2(k+1)}<2^{n}$ holds provided $n$ is sufficiently large. This proves (5.1).

In the rest of the proof let $n$ be sufficiently large to satisfy (5.1). Associated with $f \in \mathcal{F}_{n}$ satisfying (5.1), we define

$$
u(x):=\operatorname{Re}(f(x w)), \quad x \in \mathbb{R},
$$


and

$$
v(x):=\operatorname{Im}(f(x w)), \quad x \in \mathbb{R} .
$$

Obviously

$$
\left\|u^{(k+1)}\right\|_{[0,1]} \leq n^{k+2} \quad \text { and } \quad\left\|v^{(k+1)}\right\|_{[0,1]} \leq n^{k+2} .
$$

Let $y \in\left[y_{0}, 1\right]$ be an arbitrary point different from each $y_{j}$. By a well-known formula for divided differences,

$$
\frac{u(y)}{\prod_{j=0}^{k}\left(y-y_{j}\right)}+\sum_{i=0}^{k} \frac{u\left(y_{i}\right)}{\left(y_{i}-y\right) \prod_{j=0, j \neq i}^{k}\left(y_{i}-y_{j}\right)}=\frac{1}{(k+1) !} u^{(k+1)}(\xi)
$$

for some $\xi \in\left[y_{0}, 1\right]$.

Combining (5.1) and the two observations above, we obtain

$$
\begin{aligned}
|u(y)| \leq & \frac{1}{(k+1) !}\left|u^{(k+1)}(\xi)\right|\left|\prod_{j=0}^{k}\left(y-y_{j}\right)\right| \\
& \quad+\sum_{i=0}^{k}\left|u\left(y_{i}\right)\right|\left|\frac{\prod_{j=0}^{k}\left(y-y_{j}\right)}{\left(y_{i}-y\right) \prod_{j=0, j \neq i}^{k}\left(y_{i}-y_{j}\right)}\right| \\
& \leq \frac{1}{(k+1) !} n^{k+2} \frac{(k+1) !}{(2 n)^{k+1}}+2^{1-\sqrt{n}} \sum_{i=0}^{k} \frac{k !}{i !(k-i) !} \\
& \leq 2^{-(k+1)} n+2^{1-\sqrt{n}} 2^{k} \leq 2^{-(1 / 2) \sqrt{n}} n+2^{1-\sqrt{n}} 2^{(1 / 2) \sqrt{n}} \\
& \leq \exp \left(-c_{4} \sqrt{n}\right)
\end{aligned}
$$

with an absolute constant $c_{4}>0$. Similarly

$$
|v(y)| \leq \exp \left(-c_{4} \sqrt{n}\right) .
$$

Hence

$$
|f(y w)| \leq \sqrt{2} \exp \left(-c_{4} \sqrt{n}\right)
$$

with an absolute constant $c_{4}>0$. Since $y \in\left[y_{0}, 1\right]$ is arbitrary, we have proved that

$$
\|f\|_{\left[y_{0} w, w\right]} \leq \sqrt{2} \exp \left(-c_{4} \sqrt{n}\right),
$$

where $y_{0}=1-k /(2 n) \leq 1-\frac{1}{4} n^{-1 / 2}$ for sufficiently large $n$.

We conclude that the polynomial $g(x):=x^{n} f(x)$ satisfies $g \in \mathcal{F}_{2 n}$ and

$$
\|g\|_{[0, w]} \leq \exp \left(-c_{5} \sqrt{n}\right)
$$

with an absolute constant $c_{5}>0$.

Now the proof can be finished by a trivial modification of the proof given in the special case when subarc $A$ of the unit circle is symmetric with respect to the real line and contains 1 . 
Proof Lemma 4.7. We have

$$
\begin{aligned}
\left|b_{j}\right| & =\left|\frac{1}{j !} \frac{d^{j}}{d x^{j}}\left(p(x)(x-1)^{-k}\right)\right|_{x=0} \mid \\
& =\left|\frac{1}{j !} \sum_{m=0}^{j}\left(\begin{array}{c}
j \\
m
\end{array}\right)(-1)^{k} \frac{(k+m-1) !}{(k-1) !} p^{(j-m)}(0)\right| \\
& =\left|\sum_{m=0}^{j} \frac{(k+m-1) !}{(k-1) ! m !} \frac{1}{(j-m) !} p^{(j-m)}(0)\right|=\left|\sum_{m=0}^{j} \frac{(k+m-1) !}{(k-1) ! m !} a_{j-m}\right| \\
& =\left|\sum_{m=0}^{j}\left(\begin{array}{c}
k+m-1 \\
m
\end{array}\right) a_{j-m}\right| \leq 9\left(\begin{array}{c}
k+j \\
k
\end{array}\right) \leq 9\left(\frac{e(k+j)}{k}\right)^{k} \\
& \leq 9\left(\frac{e n}{k}\right)^{k}
\end{aligned}
$$

which proves the lemma.

Proof of Theorem 3.4. Without loss of generality we may assume that $A$ is the subarc of the unit circle with length $\ell(A)=a$ which is symmetric with respect to the real line and contains 1 . Let $k:=\left\lfloor\frac{2}{9 a}\right\rfloor+1$. Let $h \in \mathcal{P}_{k}$ be the polynomial with the properties of Lemma 4.8. Let $u:=k^{2}$. Let

$$
Q_{u}(x):=h^{k}(x)=: \sum_{j=0}^{u} b_{j} x^{j} .
$$

Since

$$
\begin{gathered}
|h(z)|<\exp \left(\frac{2}{k}\right), \quad|z| \leq 1, \\
\sum_{j=0}^{u}\left|b_{j}\right|^{2}=\left\|Q_{u}\right\|_{L_{2}(\partial D)}^{2} \leq \exp \left(\frac{4 k}{k}\right)=e^{4} .
\end{gathered}
$$

So

$$
\left|b_{0}\right|=1, \quad\left|b_{j}\right| \leq e^{2} \leq 9, \quad j=0,1, \ldots, u .
$$

Now let $A$ denote the subarc of the unit circle which is symmetric to the real line, contains 1 , and has length $a \in\left(0, \frac{1}{9}\right)$. Since $2 k /(9 u)=2 /(9 k) \geq a$, Lemma 4.7 implies that

$$
\left\|Q_{u}\right\|_{A} \leq \exp \left(-c_{4} k\right) \leq \exp \left(\frac{-c_{5}}{a}\right)
$$

with some absolute constants $c_{4}>0$ and $c_{5}>0$. Now let $M \geq 1$. Without loss of generality we may assume that $M=9^{m}$ with a nonnegative integer $m$. When $m=0$ the theorem follows from Theorem 3.3. So let $m \geq 1$. Let $n:=u m$ and let

$$
P_{n}(x):=Q_{u}^{m}(x):=h^{k m}(x)=: \sum_{j=0}^{n} a_{j} x^{j} .
$$

Since

$$
|h(z)|<\exp \left(\frac{2}{k}\right), \quad|z| \leq 1,
$$




$$
\sum_{j=0}^{n}\left|a_{j}\right|^{2}=\left\|P_{n}\right\|_{L_{2}(\partial D)}^{2} \leq \exp \left(\frac{4 k m}{k}\right)=e^{4 m} .
$$

So

$$
\left|a_{0}\right|=1, \quad\left|a_{j}\right| \leq e^{2 m} \leq M, \quad j=0,1, \ldots, n .
$$

Also, using $m \geq 1$, we obtain

$$
\left\|P_{n}\right\|_{A}=\left\|Q_{u}\right\|_{A}^{m}=\exp \left(\frac{-c_{5} m}{a}\right) \leq \exp \left(\frac{-c_{2}(1+\log M}{a}\right)
$$

with an absolute constant $c_{2}>0$. This finishes the proof.

Proof of Lemma 4.9. The proof is the same as that of Lemma 4.1 with trivial modifications.

Proof of Lemma 4.10. Applying Cauchy's integral formula with

$$
G(z):=g\left(z_{0}+\left(z-z_{0}\right)\right) g\left(z_{0}-\left(z_{0}-z\right)\right)
$$

on $J$, we obtain

$$
\begin{aligned}
\left|g\left(z_{0}\right)\right|^{2} & =\left|G\left(z_{0}\right)\right|=\left|\frac{1}{2 \pi i} \int_{J} \frac{G(z) d z}{z-z_{0}}\right| \\
& =\frac{2}{2 \pi}\left|\int_{J_{1}} \frac{G(z) d z}{z-z_{0}}\right| \leq \frac{1}{\pi} \int_{J_{1}} \frac{|G(z)||d z|}{\left|z-z_{0}\right|} \\
& =\frac{1}{\pi} \int_{J_{1}} \frac{\left|g\left(z_{0}+\left(z-z_{0}\right)\right) g\left(z_{0}-\left(z-z_{0}\right)\right)\right||d z|}{\left|z-z_{0}\right|} \\
& \leq(\pi \delta)^{-1} K \int_{J_{1}}|g(z)||d z| .
\end{aligned}
$$

Proof of Theorem 3.5. Without loss of generality we may assume that the $\operatorname{arc} A$ is the subarc of the unit circle with length $\ell(A)=a<\pi / 2$ which is symmetric with respect to the real line and contains 1 . Suppose $f \in \mathcal{S}_{M}^{\mu}$ and

$$
\left|f\left(\frac{1}{4 M 2^{\mu}}-\frac{1-\cos (a / 4)}{2 M 2^{\mu}}\right)\right| \geq \frac{1}{2} .
$$

Note that this is guaranteed by the assumption of the theorem since

$$
0 \leq \frac{1}{4 M 2^{\mu}}-\frac{1-\cos (a / 4)}{2 M 2^{\mu}} \leq \frac{1}{4 M 2^{\mu}},
$$

Let the region $H_{a, M, \mu}$ be defined by

$$
H_{a, M, \mu}:=\left\{z=r e^{i \theta}: \cos (a / 4)<r<1-\frac{1-\cos (a / 4)}{M 2^{\mu}},-\frac{a}{4}<\theta<\frac{a}{4}\right\} .
$$


Let $\Gamma_{a, M, \mu}$ be the circle as in Lemma 4.9. It is a simple geometric argument to show that the arc $I:=\Gamma_{a, M, \mu} \cap H_{a, M, \mu}$ has length greater than $c_{5} a$ with an absolute constant $c_{5}>0$. Let $z_{0} \in I \subset H_{a, M, \mu}$ be fixed. Then we can choose $w_{1} \in A$ and $w_{2} \in A$ such that $z_{0}=\frac{1}{2}\left(w_{1}+w_{2}\right)$. Let $J_{1}$ be the arc connecting $w_{1}$ and $w_{2}$ on the unit circle. Note that $J_{1}$ is a subarc of $A$. Let $J_{2}$ be the arc which is the symmetric image of $J_{1}$ with respect to the line segment connecting $w_{1}$ and $w_{2}$. Let

$$
g(z):=\left(\left(z-w_{1}\right)\left(z-w_{2}\right)\right)^{\mu} f(z) .
$$

Then it is elementary geometry again to show that

$$
|g(z)| \leq \frac{M\left|\left(z-w_{1}\right)\left(z-w_{2}\right)\right|^{\mu}}{(1-|z|)^{\mu}} \leq \frac{M 2^{\mu}}{\sin ^{\mu}(a / 2)}, \quad z \in J_{2} .
$$

By Lemma 4.10 we obtain

$$
\left|g\left(z_{0}\right)\right|^{2} \leq\left(\frac{\pi(1-\cos (a / 4))}{M 2^{\mu}}\right)^{-1} \frac{M 2^{\mu}}{\sin ^{\mu}(a / 2)} \int_{J_{1}}|g(z)||d z| .
$$

Observe that $f \in \mathcal{S}_{M}^{\mu}$ implies $g \in \mathcal{S}_{M 4^{\mu}}^{\mu}$. Also, since $M \geq 1$,

$$
\begin{aligned}
\left|g\left(\frac{1}{4 M 2^{\mu}}-\frac{1-\cos (a / 4)}{2 M 2^{\mu}}\right)\right| & \geq\left(1-2^{-\mu-2}\right)^{2 \mu}\left|f\left(\frac{1}{4 M 2^{\mu}}-\frac{1-\cos (a / 4)}{2 M 2^{\mu}}\right)\right| \\
& \geq\left(1-\frac{1}{8 \mu}\right)^{2 \mu}\left|f\left(\frac{1}{4 M 2^{\mu}}-\frac{1-\cos (a / 4)}{2 M 2^{\mu}}\right)\right| \\
& \geq\left(\frac{7}{8}\right)^{2} \frac{1}{2} \geq \frac{1}{4} .
\end{aligned}
$$

Hence Lemma 4.9 can be applied with $g \in \mathcal{S}_{M 4^{\mu}}^{\mu}$. We conclude that there is a point $z_{0} \in I \subset H_{a, M, \mu}$ such that

$$
\left|g\left(z_{0}\right)\right| \geq \exp \left(\frac{-c_{4}(\mu+\log M)}{a}\right) .
$$

Combining this with (5.2) and $J_{1} \subset A$ gives

$$
\begin{aligned}
\|f\|_{L_{1}(A)} & \geq\left(\frac{1}{4}\right)^{\mu}\|g\|_{L_{1}(A)} \geq\left(\frac{1}{4}\right)^{\mu}\|g\|_{L_{1}\left(J_{1}\right)} \\
& \geq\left(\frac{1}{4}\right)^{\mu} \frac{\pi(1-\cos (a / 4))}{M 2^{\mu}} \frac{\sin ^{\mu}(a / 2)}{M 2^{\mu}}\left|g\left(z_{0}\right)\right|^{2} \\
& \geq \exp \left(\frac{-c_{1}(\mu+\log M)}{a}\right)
\end{aligned}
$$

with an absolute constant $c_{1}>0$.

Proof of Corollary 3.6. Let $f \in \mathcal{K}_{M}^{\mu}$. Then $f \in \mathcal{S}_{M(\mu !)^{2}}^{\mu}$ and $f$ is continuous on the closed unit disk. Also, if $\left|z_{0}\right| \leq \frac{1}{4 M(\mu !)^{2} 2^{\mu}}$, then

$$
\begin{aligned}
\mid f\left(z_{0} \mid\right. & \geq 1-M(\mu !)^{2} \sum_{j=1}^{\infty} j^{\mu}\left(\frac{1}{4 M(\mu !)^{2} 2^{\mu}}\right)^{j} \geq 1-\frac{M(\mu !)^{2}}{4 M(\mu !)^{2}} \sum_{j=1}^{\infty}\left(\frac{j}{2^{j}}\right)^{\mu} \\
& \geq 1-\frac{1}{4} \sum_{j=1}^{\infty}\left(\frac{j}{2^{j}}\right) \geq 1-\frac{2}{4} \geq \frac{1}{2} .
\end{aligned}
$$


So the assumptions of Theorem 3.5 are satisfied with $M$ replaced by $M(\mu !)^{2}$, and the corollary follows from Theorem 3.5.

Proof of Corollary 3.7. Let

$$
p_{k}(z)=\sum_{j=0}^{n_{k}} a_{j, k} z^{j}, \quad a_{j, k} \in \mathbb{C}, \quad a_{n_{k}, k} \neq 0,
$$

and let $M_{k}:=H\left(p_{k}\right)$. Applying Corollary 3.6 with

$$
q_{k}(z):=\left|a_{n_{k}, k}\right|^{-1} z^{n_{k}} p_{k}\left(z^{-1}\right) \in \mathcal{K}_{M_{k}}\left(=\mathcal{K}_{M_{k}}^{1}\right)
$$

and the $\operatorname{arc} B:=\left\{z^{-1}: z \in A\right\}$ of length $a$, we obtain the corollary.

\section{Proof of Theorem 3.8}

Lemma 6.1. For every $r \in(0,1 / 2)$ there exists a trigonometric polynomial

$$
p(z)=\sum_{j=-n}^{n} c_{j} z^{j}
$$

such that $c_{0}=1,\left|c_{j}\right|<r$ and $|p(z)|<r$ everywhere on the unit circle except possibly in a set of linear measure at most $r$.

Proof. The finite Riesz product

$$
p(z)=\prod_{j=1}^{N}\left(1+r z^{m_{j}}+r z^{-m_{j}}\right)
$$

with $m_{j}:=4^{j}$ and sufficiently large $N$ is such an example. For $r \in(0,1 / 2)$ and $m_{j}=4^{j}$ the Riesz products tend to 0 almost everywhere on the unit circle as $N \rightarrow \infty$. See, for example, [Zy-59, p 208].

The next lemma follows simply from the fact that the transfinite diameter of any closed proper subset of the unit circle is less than 1 . (We remark that due to this fact the polynomial guaranteed by Lemma 6.2 can be chosen so that its coefficients are integers. We will not need this extra property.)

Lemma 6.2. For every $\eta>0$ there exists a polynomial

$$
g(z)=\sum_{k=0}^{L} b_{k} z^{k}
$$

such that $b_{0}=1$ and $|g(z)|<\eta$ everywhere on the unit circle except possibly on a set of linear measure at most $\eta$. 
Proof of Theorem 3.8. For $\eta:=\epsilon / 2$ we choose a polynomial

$$
g(z)=\sum_{k=0}^{L} b_{k} z^{k}
$$

with the properties of Lemma 6.2 , that is, $b_{0}=1$ and

$$
\begin{aligned}
& |g(z)|<\frac{\epsilon}{2} \quad \text { everywhere on the unit circle except } \\
& \text { possibly in a set of linear measure at most } \frac{\epsilon}{2} .
\end{aligned}
$$

For every $k$ with $\left|b_{k}\right|>1$ we choose a trigonometric polynomial

$$
p_{k}(z)=\sum_{j=-n_{k}}^{n_{k}} c_{j, k} z^{j}
$$

so that

$$
c_{0, k}=1, \quad\left|c_{j, k}\right|<\frac{\epsilon}{2 L\left|b_{k}\right|}
$$

and

$$
\begin{aligned}
& \left|p_{k}(z)\right|<\frac{\epsilon}{2 L\left|b_{k}\right|} \quad \text { everywhere on the unit circle except } \\
& \text { possibly in a set of linear measure at most } \frac{\epsilon}{2 L} .
\end{aligned}
$$

This can be done by Lemma 6.2 . Now let

$$
h(z):=g\left(z^{A}\right)=\sum_{k=0}^{L} b_{k} z^{A k} \quad \text { with } \quad A:=1+2 \max _{k:\left|b_{k}\right|>1} n_{k} .
$$

Finally we define

$$
f(z):=h(z)-\sum_{\substack{k=1 \\\left|b_{k}\right|>1}}^{L} b_{k} z^{A k} p_{k}(z) .
$$

It is straightforward from the construction that $f \in \mathcal{K}_{1}$. Also, (6.1) and the definition of $h$ imply that

$$
\begin{aligned}
& |h(z)|<\frac{\epsilon}{2} \quad \text { everywhere on the unit circle except } \\
& \text { possibly in a set of linear measure at most } \frac{\epsilon}{2} \text {. }
\end{aligned}
$$

Finally (6.2) and the definition of $f$ imply that

$$
|f(z)|<\frac{\epsilon}{2}+\sum_{\substack{k=1 \\\left|b_{k}\right|>1}}^{L}\left|b_{k}\right| \frac{\epsilon}{2 L\left|b_{k}\right|} \leq \frac{\epsilon}{2}+L \frac{\epsilon}{2 L}=\epsilon
$$

everywhere on the unit circle except possibly in a set of linear measure at most

This finishes the proof.

$$
\frac{\epsilon}{2}+L \frac{\epsilon}{2 L}=\epsilon \text {. }
$$

\section{Acknowledgment.}

We thank Fedor Nazarov for observing and proving Theorem 3.8. The content of Section 6 is due to him. We also thank him for several discussions about problems related to the paper. 


\section{REFERENCES}

Be-95. J. Beck, Flat polynomials on the unit circle - note on a problem of Littlewood, Bull. London Math. Soc. 23 (1991), 269-277.

Bou-86. J. Bourgain, Sul le minimum d'une somme de cosinus, Acta Arith. 45 (1986), 381-389.

Bor-95. P. Borwein and T. Erdélyi, Polynomials and Polynomial Inequalities, Springer-Verlag, New York, 1995.

Bor-96. P. Borwein and T. Erdélyi, Littlewood-type problems on $[0,1]$, manuscript.

Ka-85. J-P. Kahane, Sur les polynômes á coefficients unimodulaires, Bull. London Math. Soc 12 (1980), 321-342.

Ko-81. S. Konjagin, On a problem of Littlewood, Izv. A. N. SSSR, ser. mat. 45, 2 (1981), 243-265.

Ko-96. S. Konjagin, On a question of Pichorides (to appear).

Li-68. J.E. Littlewood, Some Problems in Real and Complex Analysis, Heath Mathematical Monographs, Lexington, Massachusetts, 1968.

Ma-63. K. Mahler, On two extremal properties of polynomials, Illinois J. Math. 7 (1963), 681701.

Na-93. F.L. Nazarov, Local estimates of exponential polynomials and their applications to the inequalities of uncertainty principle type, St. Petersburg Math. J. 5 (1994), 663-717.

Ne-90. D.J. Newman and J. S. Byrnes, The $L^{4}$ norm of a polynomial with coefficients \pm 1 , MAA Monthly 97 (1990), 42-45.

Od-93. A. Odlyzko and B. Poonen, Zeros of polynomials with 0,1 coefficients, Ens. Math. 39 (1993), 317-348.

Pi-83. S.K. Pichorides, Notes on trigonometric polynomials., in: Conference on harmonic analysis in honor of Antoni Zygmund, Vol. I, II (Chicago, Ill., 1981), 84-94, Wadsworth Math. Ser., Wadsworth, Belmont, Calif., 1983..

MPS-81. O.C. McGehee, L. Pigno and B. Smith, Hardy's inequality and the $L^{1}$-norm of exponential sums, Ann. Math. 113 (1981), 613-618..

So-95. B. Solomyak, On the random series $\sum \pm \lambda^{n}$ (an Erdös problem), Annals of Math. 142 (1995), 611-625.

Tu-84. P. Turán, On a New Method of Analysis and its Applications, Wiley, New York, 1984.

Zy-59. A. Zygmund, Trigonometric Series, Vol. 2, Cambridge University Press, New York, 1959.

Department of Mathematics and Statistics, Simon Fraser University, Burnaby, B.C., Canada V5A 1S6 (pborwein@CeCm.Sfu.Ca)

Department of Mathematics, Texas A\&M University, College Station, Texas 77843 (TERDELYi@MATH.TAMU.EDU) 